\title{
Drivability Analysis of Through-the-Road-Parallel Hybrid Vehicles
}

\author{
E. Galvagno - D. Morina - A. Sorniotti · M. Velardocchia
}

Received: date / Accepted: date

\begin{abstract}
In the last decade, Hybrid Electric Vehicles (HEVs) have spread worldwide due to their capability to reduce fuel consumption. Several studies focused on the optimisation of the energy management system of hybrid vehicles are available in literature, whilst there are few articles dealing with the drivability and the dynamics of these new powertrain systems. In this paper a 'Through-the-Road-Parallel HEV' is analysed. This architecture is composed of an internal combustion engine mounted on the front axle and an electric motor powering the rear one. These two powertrains are not directly connected to each other, as the parallel configuration is implemented through the roadtyre force interaction. The main purpose of this paper is the drivability analysis of this layout of HEVs, using linearised mathematical models in both time (i.e. vehicle response during tip-in tests) and frequency domain (i.e. frequency response functions), considering the effect of the engaged gear ratio. The differences
\end{abstract}

\section{E. Galvagno}

Politecnico di Torino - Dipartimento di Meccanica,

Corso Duca degli Abruzzi, 24,10129, Torino, ITALY

E-mail: enrico.galvagno@polito.it

D. Morina

Politecnico di Torino - Dipartimento di Meccanica,

Corso Duca degli Abruzzi, 24,10129, Torino, ITALY

E-mail: morina.dario@gmail.com

A. Sorniotti

University of Surrey, Faculty of Engineering and Physical Sciences, Guildford, Surrey, GU2 7XH, UK

E-mail: a.sorniotti@surrey.ac.uk

M. Velardocchia

Politecnico di Torino - Dipartimento di Meccanica,

Corso Duca degli Abruzzi, 24,10129,Torino, ITALY

E-mail: mauro.velardocchia@polito.it from a traditional Front-Wheel-Drive (FWD) configuration are subsequently highlighted. Furthermore, the authors compare different linearised dynamic models, with an increasing number of degrees of freedom, in order to assess which model represents the best compromise between complexity and quality of the results. Finally, a sensitivity analysis of the influence of the torque distribution between the front (thermal) and rear (electric) axles on vehicle drivability is carried out and presented in detail.

Keywords drivability · hybrid electric vehicle · linear powertrain model · sprung mass dynamics · state space - vehicle pitch and shake motions - frequency response function · tip-in.

\section{Introduction}

Low frequency drivability $(1-15 \mathrm{~Hz})$ is an important aspect when evaluating vehicle dynamic performance; it describes the longitudinal dynamics of a vehicle in response to driver inputs in a comprehensive range of driving situations, and the driver subjective perception of that behaviour. In order to effectively analyse vehicle drivability in conditions of constant gear ratio, linearised models can generally be adopted, whilst more complex non-linear dynamic models are required for the assessment of gearshift quality and its impact on drivability. The focus of this paper is limited to the conditions of constant gear ratios on both axles of 'Through-theRoad-Parallel HEVs', as the detailed analysis of the gearshift phase would require the models of the actuation system and transmission control algorithm. In order to obtain the natural frequencies, modal shapes and frequency response functions of the overall system, the equations of the implemented models are linearised. 
The first step towards the evaluation of vehicle drivability is the research into a correlation between the subjective rating of vehicle performance, carried out by the passengers and driver, and objective parameters and indices that can be defined and computed starting from measurable variables. Both tip-in and gearshift manoeuvres were analysed in detail for FWD sedans by Dorey [5] and Sorniotti [11]. The main conclusion is that ride comfort is strictly correlated with the time history of vehicle longitudinal acceleration, i.e. overshoot and rise rate, in case of tip-in, and with the torque and acceleration gap, in case of gearshift. Also vehicle jerk, the time derivative of the longitudinal acceleration, is an important index of vehicle comfort, and can be easily correlated with the subjective evaluations [3]. Choi et al. [4], through the experimental study of the tip-in maneouvre, demonstrates that the typical shuffle dynamics of internal combustion engine driven vehicles are caused by sudden variations of engine torque and that their frequency is a function of the engaged gear ratio. Moreover, the moment of inertia of engine and flywheel is highlighted as a key factor for the shuffle characteristics. Mathematical models to understand and evaluate the influence of the driveline components on vehicle longitudinal dynamics have been implemented in [12]. The paper discusses the need to use non-linear models, including the characteristics of the clutch torsional damper and tyres, to study the dynamics of internal-combustion-engine-driven powertrains in the time domain. Furthermore, in the same paper, a detailed study of linearised models in the frequency domain has been dealt with. In order to obtain realistic results, it is essential to remove the pure rolling hypothesis in the tyre-road interaction, and to introduce an additional damping for modelling slip ratio dynamics. The first natural frequency of a conventional driveline increases as a function of the engaged gear, and it is located in the $2-10 \mathrm{~Hz}$ range. The frequencies associated to the dynamics of vehicle sprung mass are relatively low compared with the driveline frequencies, whilst the frequencies associated to the engine mounting system are comparable with the drivetrain frequencies.

In order to minimise driveline oscillations in conventional vehicles while retaining fast acceleration, so-called anti-jerk controls have been developed. The output variable of these control systems is a correction torque subtracted from the torque requested by the driver. In [3] a robust controller design based on a state space model is dealt with. The H-infinity approach guarantees the stability of the control loop against the variation of the main parameters of the system, e.g. due to the wear of the components. In [13], on the other hand, an anti-jerk controller based on Neuro-Fuzzy models is presented; a root-locus method with pole-placement is applied for the controller design.

Finally, the effect of asymmetrical geometry and compliance between the left and right side of the vehicle are dealt with by B.S. Kim et al. [7], where it is demonstrated that during acceleration half-shaft stiffness is one of the most important factor in vehicle drift.

With the introduction of the HEVs in the automotive market, significant experimental testing activities have been performed on this new type of vehicle. The reasons why hybrid electric powertrains produce jerk are different when compared with a traditional internal combustion engine configuration. In HEVs, driveline oscillations can mainly occur during the following phases: starting, switching of operation mode, shifting and regenerative braking [14]. A way of simultaneously controlling the internal combustion engine and the electric motor to actively damp driveline oscillations is presented in [6]. In [8] a transient control for EV/HEV mode changes is presented, based on clutch slip control methods.

In conclusion, the authors have found few publications about hybrid vehicle drivability in conditions of engaged gear and a study in the frequency domain for the hybrid through-the-road powertrain layout is absent at the present time.

The purpose of this study is to assess the drivability of a through-the-road-parallel HEV, with an internal combustion engine powering the front axle and an electric motor the rear axle. Six dynamic models characterised by increasing complexity are presented with the aim of identifying the best trade-off between model sophistication and adequate results. The most complex model is described in detail. The mathematical model presented in the paper includes both driveline and vehicle dynamics. More specifically, the driveline model considers the effect of the asymmetry (e.g. half-shafts with different stiffness) between the left and right side of the vehicle. The vehicle model allows the assessment of sprung and unsprung mass dynamics, considering pitching and shaking motions. By using this linearised model, simulations can be carried out in the time domain and frequency domain by examining the natural frequencies of the system, drawing frequency response functions and studying the modal shapes. In such a way, it is possible to evaluate the drivability of the hybrid vehicle and compare it with a conventional FWD internal-combustion-engine-driven vehicle considering also the effect of the single model parameters on the overall dynamic performance. 


\section{Linear dynamic models}

These models do not consider the powertrain mounting system, because the mounting system dynamics are generally highly damped and therefore do not significantly affect vehicle response.

In particular, the main features of these models sorted by complexity are:

- Simple model (see Fig. 2): ideal engine and electric motor, i.e. no time delay between the requested and the actuated torque, locked differentials, one front and one rear equivalent half-shaft, linearised steadystate Pacejka model [9] for the two equivalent tyres, rigid suspension system and vehicle purely longitudinal dynamics;

- Relaxation length model: the effect of tyre relaxation length is added to each equivalent wheel of the previous model;

- Engine and electric motor delay model: first order dynamics are added both for the engine and electric motor in order to take into account the time-delays introduced by the respective torque actuation systems;

- Half-shaft model: all four half-shafts are modelled independently thus allowing investigation into the effect of different half-shaft stiffness and damping parameters, whilst the differentials are still considered locked;

- Open differential model: front and rear differentials are modelled as 'open differentials', considering both their inertial and kinematic properties;

- Vehicle sprung and unsprung mass model (see Fig. 3): the vehicle sprung mass and unsprung mass dynamics replace the one degree-of-freedom (DOF) purely longitudinal dynamics of a rigid chassis without suspension used for all the previous models. In this way, also the vertical displacement and the pitch angle of the chassis are considered together with the horizontal and vertical positions of the front and rear wheel hubs.

With reference to the most complex model configuration, a schematic representation of the driveline and the vehicle model is depicted in Fig. 3, where points $O_{1}$, $\mathrm{O}_{2}, \mathrm{O}_{3}$ and $\mathrm{O}_{4}$ are the connection between the two systems, achieved through the unsprung masses, while the powertrain is considered rigidly coupled to the chassis, so the dynamics of its mounting system is neglected. The suspension characteristics introduced in this model are the equivalent trailing arm suspension properties that can be obtained from any suspension type [10]: suspension vertical stiffness (front: $K_{s, f}$, rear: $K_{s, r}$ ), suspension vertical damping (front: $\beta_{s, f}$, rear: $\beta_{s, r}$ ) and position of the trailing arm attachment $(c, e, d, n)$. The relative displacements between the sprung mass and the the unsprung masses are obtained through the geometric relationships of the suspension. The overall system in Fig. 3 is characterised by 16 DOFs: one for the engine and the clutch $\left(\vartheta_{e}\right)$, one for front gearbox and front differential case $\left(\vartheta_{d f, f}\right)$; two for the differentials, one for the speed difference between front sun gears $\left(\Delta \vartheta_{s, f}\right)$ and one for the rear ones $\left(\Delta \vartheta_{s, r}\right)$; four for the wheels $\left(\vartheta_{w, f, L}, \vartheta_{w, f, R}, \vartheta_{w, r, L}\right.$ and $\left.\vartheta_{w, r, R}\right)$; one for the electric motor, the rear gearbox and the rear differential case $\left(\vartheta_{m}\right)$; three for the sprung mass (longitudinal $x_{s m}$ and vertical $z_{s m}$ displacement and pitch angle $\vartheta_{s m}$ ); four for the vertical position of the unsprung front and rear masses $\left(z_{u s, f, R}, z_{u s, f, L}, z_{u s, r, R}\right.$ and $\left.z_{u s, r, L}\right)$. The dimension of the dynamic matrix is $38 \times 38$ : two times the number of DOFs which is further increased by four for the tyre delayed torques, one for engine delayed torque and one for electric motor delayed torque. Consequently, the state vector $z$ can be written as follows:

$\mathbf{z}_{38 \times 1}=\left\{\begin{array}{c}\dot{\vartheta}_{e} \\ \dot{\vartheta}_{d f, f} \\ \dot{\vartheta}_{w, f, R} \\ \dot{\vartheta}_{w, f, L} \\ \dot{\vartheta}_{w, r, R} \\ \dot{\vartheta}_{w, r, L} \\ \dot{\vartheta}_{m} \\ \Delta \dot{\vartheta}_{s, f} \\ \Delta \dot{\vartheta}_{s, r} \\ \dot{x}_{s m} \\ \cdots\end{array}\right\}\left\{\begin{array}{c}\ldots \\ \dot{z}_{s m} \\ \dot{\vartheta}_{s m} \\ \dot{z}_{u s, f, R} \\ \dot{z}_{u s, f, L} \\ \dot{z}_{u s, r, R} \\ \dot{z}_{u s, r, L} \\ \vartheta_{e} \\ \vartheta_{d f, f} \\ \vartheta_{w, f, R} \\ \vartheta_{w, f, L} \\ \cdots\end{array}\right\}\left\{\begin{array}{c}\ldots \\ \vartheta_{w, r, R} \\ \vartheta_{w, r, L} \\ \vartheta_{m} \\ \Delta \vartheta_{s, f} \\ \Delta \vartheta_{s, r} \\ x_{s m} \\ z_{s m} \\ \vartheta_{s m} \\ z_{u s, f, R} \\ z_{u s, f, L} \\ \ldots\end{array}\right\}\left\{\begin{array}{c}\ldots \\ z_{u s, r, R} \\ z_{u s, r, L} \\ T_{d t, f, R} \\ T_{d t, f, L} \\ T_{d t, r, R} \\ T_{d t, r, L} \\ T_{d e} \\ T_{d m}\end{array}\right\}$

In the following section the dynamic equations of the driveline and vehicle body components will be detailed in order to write the state-space matrices.

\subsection{Dynamic equations}

The dynamic equations can be written by dividing them into two groups: those of the driveline and those of the vehicle.

\subsubsection{Nomenclature}

The nomenclature adopted in the following equations is given in Fig. 1: the capital letter $A$ indicates the main term, while the following subscripts $(b, c$ and $d)$ are used to better specify its meaning. 


A - main letter:
$\beta$ : damping
F: force
$\eta$ : gear efficienty
i: gear ratio
J: inertia
K: stiffness
m: mass
$\theta:$ angle
$\dot{\theta}:$ rotation velocity
$\ddot{\theta}:$ rotation acceleration
R: radius
T: torque
$\tau:$ constant delay
x: longitudinal displacement
$\dot{x}:$ longitudinal velocity
$\ddot{x}:$ longitudinal acceleration
z: vertical displacement
$\dot{z}:$ vertical velocity
$\ddot{z}:$ vertical acceleration

b - first subscript:

aer: aerodynamic

c: clutch

cd: cutch damper

de: delay engine

df: differential

$\mathrm{dm}$ : delay electric motor

$\mathrm{dt}$ : delay tyre

e: engine

g: gear

g1: primary gear shaft

g2: secondary gear shaft

hs: half-shaft

$\mathrm{j}$ : suspension joint

$\mathrm{m}$ : electric motor

p: planetary gear

roll: rolling

s: sungear

sm: spung mass

su: suspension

t: tyre

us: unsprung mass

w: wheel
A

$b, c, d$

$\uparrow \uparrow \uparrow_{\text {second subscript }}$ third subscript

first subscript

main letter

c - second subscript:

0 : initial condition

3: upper planetary gear

4: lower planetary gear

f: front

L: left

r: rear

R: right

sm: spung mass

us: unsprung mass

$x$ : vertical componet

z: horizontal component

d - third subscript:

L: left

R: right

Figure 1: Nomenclature.

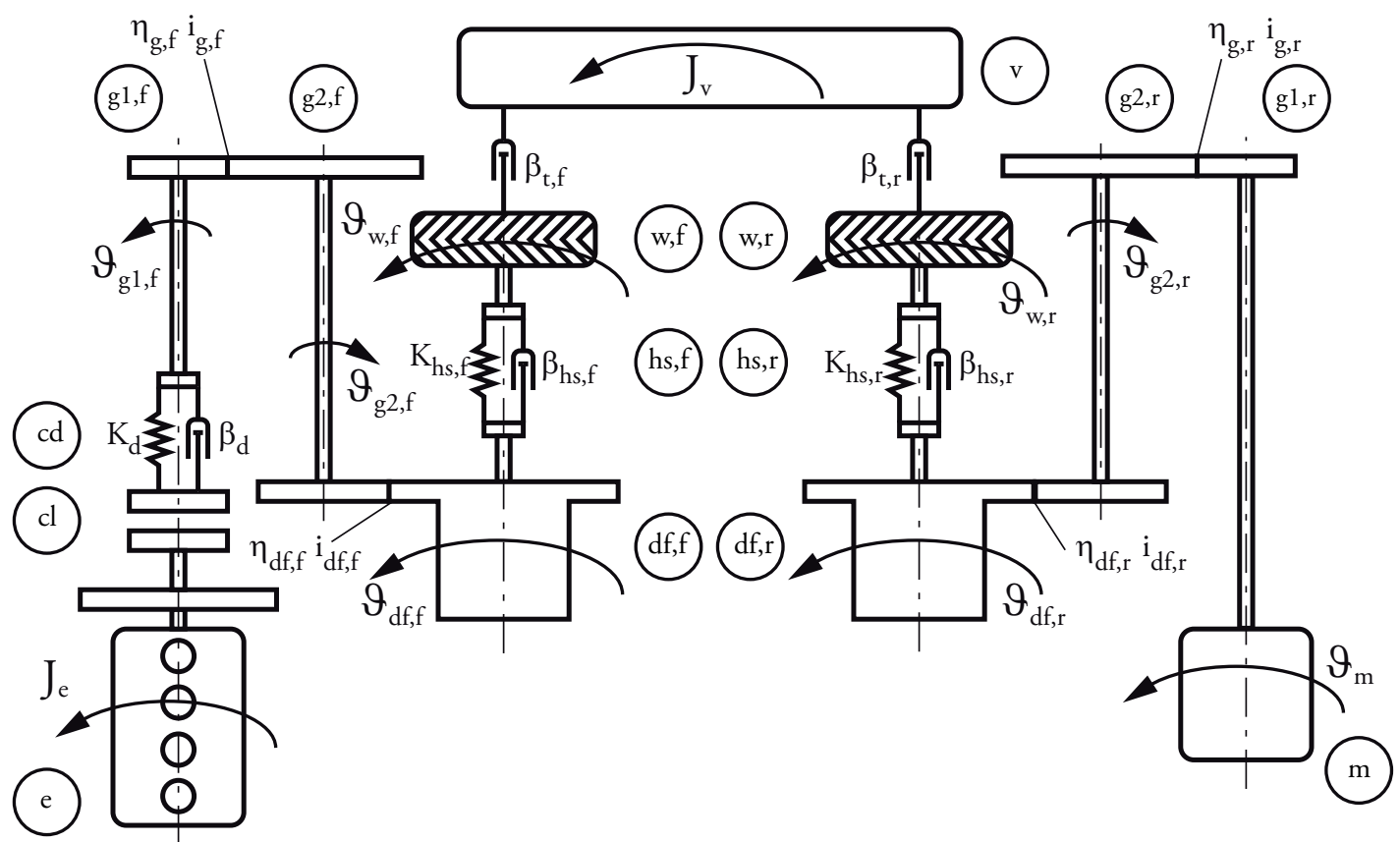

Figure 2: Through-the-road-parallel HEV - Simple Model.

$e$ : engine, $c l$ : clutch, $c d$ : clutch dumper, $g 1, f$ : front gearbox primary shaft, $g 2, f$ : front gearbox secondary shaft, $d f, f$ : front differential, $h s, f:$ front equivalent half-shaft, $w, f$ : front equivalent wheel, $d f, r:$ rear differential, $h s, r:$ rear equivalent half-shaft, $w, r$ : rear equivalent wheel, $v$ : 1 d.o.f. vehicle, $g 1, r$ : rear gearbox primary shaft, $g 2, r$ : rear gearbox secondary shaft and $m$ : electric motor. 


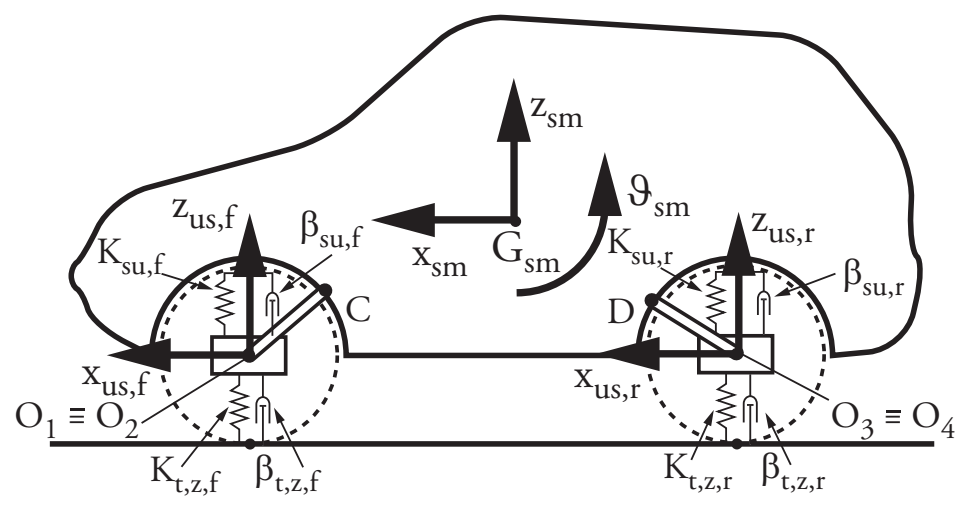

(a)

Vehicle Layout

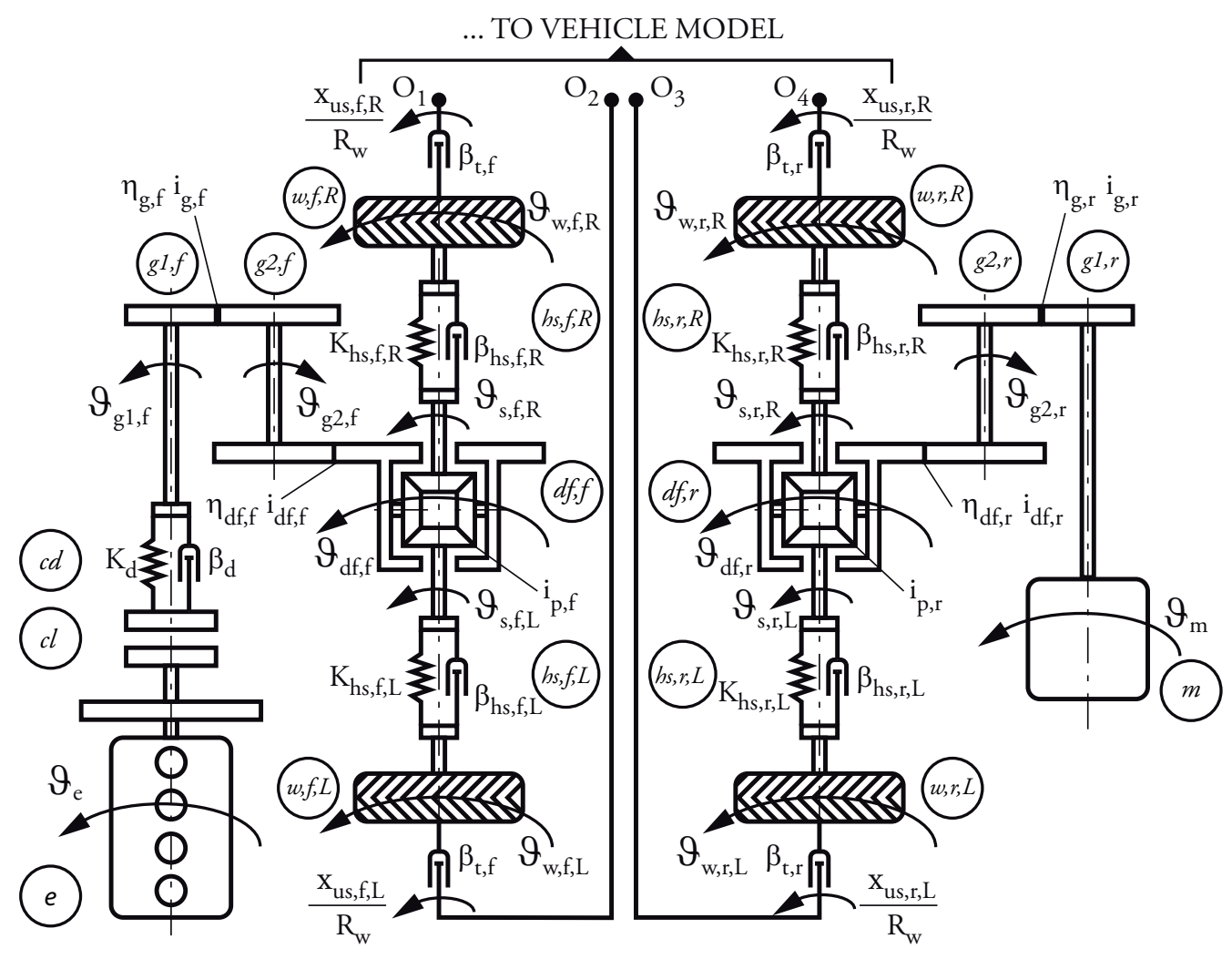

(b)

Transmission and Driveline Layout

Figure 3: Through-the-road-parallel HEV - Vehicle Sprung and Unsprung Mass Model.

$e$ : engine, $c l$ : clutch, $c d$ : clutch damper, $g 1, f$ : front gearbox primary shaft, $g 2, f$ : front gearbox secondary shaft, $w, f, L$ : front left wheel, $h s, f, L$ : front left half-shaft, $d f, f$ : front differential, $h s, f, R$ : front right half-shaft, $w, f, R$ : front right wheel, $w, r, L$ : rear left wheel, $h s, r, L$ : rear left half-shaft, $d f, r$ : rear differential, $h s, r, R$ : rear right half-shaft, $w, r, R$ : rear right wheel, $g 1, r$ : rear gearbox primary shaft, $g 1, r$ : rear gearbox secondary shaft and $m$ : electric motor.

\subsubsection{Driveline}

The driveline configuration described in the paper (see Fig. 3) considers the clutch fully engaged and fixed values of the gear ratios of the front and rear gearboxes during the tests. The engine and clutch dynamics are described in equation (2), in which $\vartheta_{e}$ is the engine de- gree of freedom.

$$
T_{d e}-\left(J_{e}+J_{c}\right) \ddot{\vartheta}_{e}-T_{c d}=0
$$

The torque transferred by the clutch torsional damper $T_{c d}$ can be modelled as the sum of an elastic term, representative of the clutch damper springs, and a viscous dissipation term, which approximates the dry friction 
effects of the clutch damper:

$T_{c d}=K_{c d}\left(\vartheta_{e}-\vartheta_{d f, f} i_{g, f} i_{d f, f}\right)+\beta_{c d}\left(\dot{\vartheta}_{e}-\dot{\vartheta}_{d f, f} i_{g, f} i_{d f, f}\right)$

The dynamic equation for the electric motor and the rear gearbox is:

$T_{d m}-\left(J_{m}+J_{g 1, r}+\frac{J_{g 2, r}}{i_{g, r}^{2} \eta_{g, r}}\right) \ddot{\vartheta}_{m}-\frac{T_{d f, r}}{i_{g, r} \eta_{g, r}}=0$

where $\vartheta_{m}$ is the electric motor degree of freedom, $i_{g, r}=$ $\dot{\vartheta}_{g 1, r} / \dot{\vartheta}_{g 2, r}$ and $i_{d f, r}=\dot{\vartheta}_{g 2, r} / \dot{\vartheta}_{d f, r}$ are the gear ratios of the rear gearbox and rear differential respectively.

Equations (2) and (4) contain the delayed torques from the engine $T_{d e}$ and from the electric motor $T_{d m}$. The actuators are modelled as first order systems in order to consider their delay; under this hypothesis the differential equations that link the demanded torques $\left(T_{m}\right.$ and $\left.T_{e}\right)$ with the delayed ones $\left(T_{d m}\right.$ and $\left.T_{d e}\right)$ are:

$T_{e}=\tau_{e} \dot{T}_{d e}+T_{d e}$

$T_{m}=\tau_{m} \dot{T}_{d m}+T_{d m}$

The time constant values $\tau_{e}$ and $\tau_{m}$ used for the two actuators are significantly different $\left(\tau_{m}<<\tau_{e}\right)$.

The front gearbox dynamic equation is:

$i_{g, f} \eta_{g, f} T_{c d}-\left(i_{g, f}^{2} \eta_{g, f} J_{g 1, f}+J_{g 2, f}\right) \ddot{\vartheta}_{g 2, f}-T_{d f, f}=0$

The free-body diagram of an open differential is depicted in Fig. 4. This component has two degrees of freedom, $\vartheta_{d f}$, i.e. the angular position of the differential case, and $\Delta \vartheta_{s}=\vartheta_{s, R}-\vartheta_{s, L}$, i.e. the difference between the rotation of the right and left sun gears.

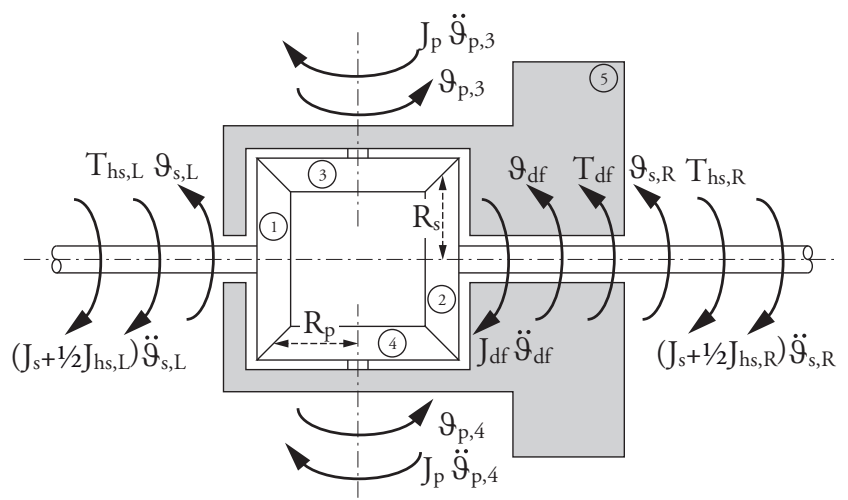

Figure 4: Differential model: (1) left sun gear, (2) right sun gear, (3) upper planet gear, (4) lower planet gear and (5) differential case.

The two dynamic equations for the open differential are:

$i_{d f} \eta_{d f} T_{d f}-J_{e q, 1} \ddot{\vartheta}_{d f}-\frac{\Delta J_{h s}}{4} \Delta \ddot{\vartheta}_{s}-\left(T_{h s, R}+T_{h s, L}\right)=0$
$\frac{\Delta J_{h s}}{2} \ddot{\vartheta}_{d f}+J_{e q, 2} \Delta \ddot{\vartheta}_{s}+T_{h s, R}-T_{h s, L}=0$

where:

$$
\begin{aligned}
& J_{e q, 1}=J_{d f}+2 J_{s}+\frac{J_{h s, R}+J_{h s, L}}{2} \\
& J_{e q, 2}=J_{s}+\frac{J_{h s, R}+J_{h s, L}}{4}+i_{p}^{2} J_{p} \\
& \Delta J_{h s}=J_{h s, R}-J_{h s, L} \\
& i_{p}=R_{s} / R_{p}
\end{aligned}
$$

The kinematic equations that link the DOFs of the differential with the speed of the two sun gears, $\dot{\vartheta}_{s, L}$ and $\dot{\vartheta}_{s, R}$, are obtained through simple mathematical steps, starting from Willis formula:

$\left\{\begin{array}{l}\dot{\vartheta}_{s, R}=\dot{\vartheta}_{d f}+1 / 2 \Delta \dot{\vartheta}_{s} \\ \dot{\vartheta}_{s, L}=\dot{\vartheta}_{d f}-1 / 2 \Delta \dot{\vartheta}_{s}\end{array}\right.$

The planet speeds are proportional to the speed difference between the sun gears $\Delta \dot{\vartheta}_{s}$ :

$\left\{\begin{array}{l}\dot{\vartheta}_{p, 3}=-i_{p} \Delta \dot{\vartheta}_{s} / 2 \\ \dot{\vartheta}_{p, 4}=i_{p} \Delta \dot{\vartheta}_{s} / 2\end{array}\right.$

In the equations (7) and (8), the half-shaft torques $T_{h s, R}$ and $T_{h s, L}$ are used; their equation is:

$T_{h s}=K_{h s}\left(\vartheta_{s}-\vartheta_{w}\right)+\beta_{h s}\left(\dot{\vartheta}_{s}-\dot{\vartheta}_{w}\right)$

The rotational dynamics of the wheels are written in equation (13), where: $\vartheta_{w}$ is the wheel DOF and $T_{\text {roll }}$ is the rolling resistance torque (that will be shown later in equation (29)).

$T_{h s}-T_{d t}-\left(J_{w}+\frac{J_{h s}}{2}\right) \ddot{\vartheta}_{w}-T_{\text {roll }}=0$

The delay between the steady-state $\left(T_{t}\right)$ and transient $\left(T_{d t}\right)$ torque generated by the tyre-road interaction is modelled using the longitudinal relaxation length $\left(L_{r}\right)$.

$T_{t}=\tau_{w} \dot{T}_{d t}+T_{d t}$

The time constant $\tau_{w}$, due to the relaxation length, is inversely proportional to the vehicle longitudinal speed $\dot{x}_{s m}$ :

$\tau_{w}=\frac{L_{r}}{\dot{x}_{u s}} \approx \frac{L_{r}}{\dot{x}_{s m_{0}}}$

It is possible to use a constant vehicle speed, e.g. $\dot{x}_{s m_{0}}$ the initial velocity, in order to obtain a linear model. However, this model has some shortcomings: at low speeds, the delay tends to infinity $\left(\dot{x}_{s m_{0}} \approx 0\right)$, and it also neglects the dependence of the relaxation length on the longitudinal slip (as explained in [9], the relaxation length decreases with increasing slip). Therefore, in order to assess this phenomenon correctly, a non-linear model would be required. 


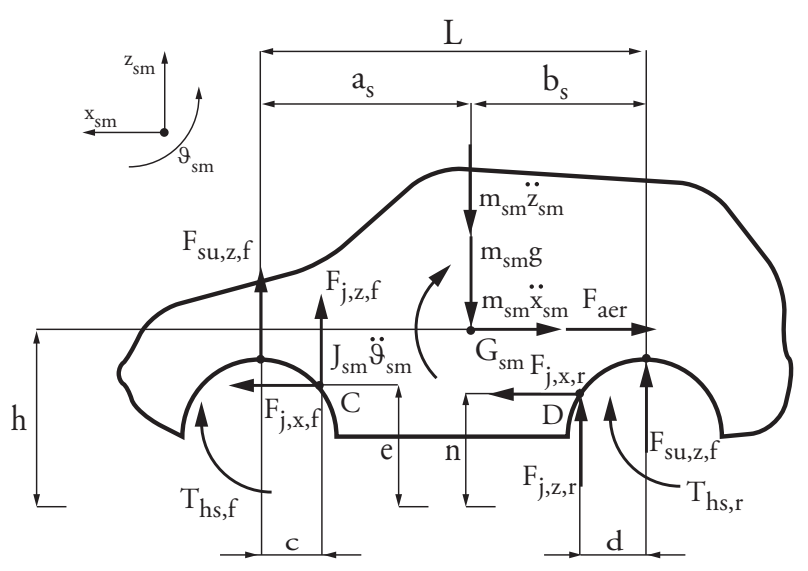

Figure 5: Free body diagram of the sprung mass.

\subsubsection{Vehicle}

The vehicle model can be decomposed into three main subsystems: the sprung mass, the front unsprung masses and the rear unsprung masses. For each of them the motion equations, starting from the respective free body diagrams, will be presented. Front and rear suspensions are modelled as equivalent trailing arm suspensions, since it is always possible to convert different layouts to this one.

The free body diagram of the sprung mass is depicted in Fig. 5, and according to that sign convention, the longitudinal and vertical force balance, equations (16) and (17) respectively, and the moment balance about the sprung mass centre of gravity $G_{s m}$, equation (18), are formulated.

$\sum_{k=f, r} F_{j, x, k}-F_{a e r}-m_{s m} \ddot{x}_{s m}=0$

where $\ddot{x}_{s m}$ is the longitudinal acceleration of the sprung mass.

$\sum_{k=f, r} F_{j, z, k}+\sum_{k=f, r} F_{s, z, k}-m_{s m} \ddot{z}_{s m}=0$

where $\ddot{z}_{s m}$ is the vertical acceleration of the sprung mass.

$$
\begin{gathered}
-F_{s, z, f} a_{s}-F_{j, z, f}\left(a_{s}-c\right)+F_{s, z, r} b_{s}+F_{j, z, r}\left(b_{s}-d\right)-F_{j, x, f} \\
(h-e)-F_{j, x, r}(h-n)-\sum_{k=f, r} T_{h s, k}-J_{s m} \ddot{\vartheta}_{s m}=0
\end{gathered}
$$

where $\vartheta_{s m}$ is the rotational DOF of the sprung mass, i.e. the vehicle pitch angle.

The vertical suspension forces generated by the springs

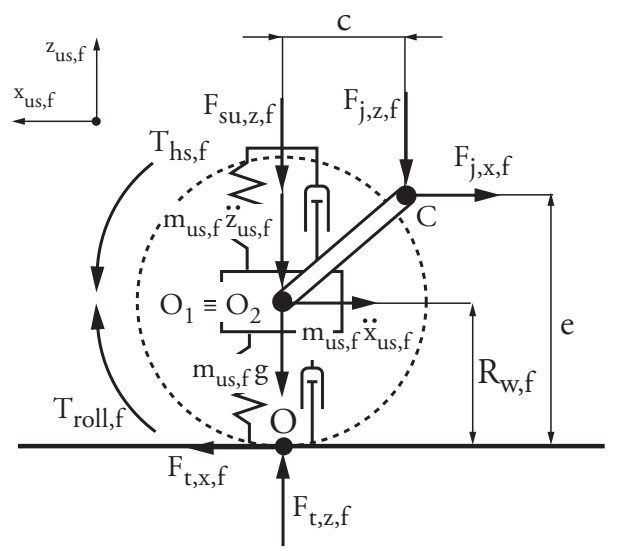

(a)

Front

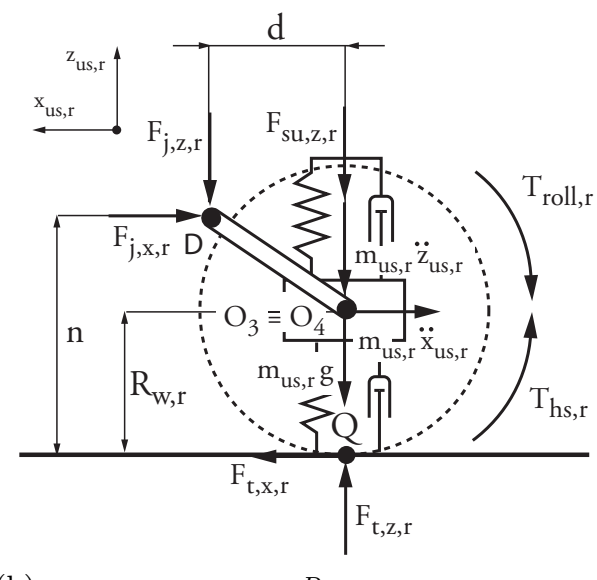

(b)

$$
\text { Rear }
$$

Figure 6: Free body diagrams of the unsprung masses.

and dampers $\left(F_{s, z, f, R}, F_{s, z, f, L}, F_{s, z, r, R}\right.$ and $\left.F_{s, z, r, L}\right)$ can be written as:

$F_{s, z, f}=K_{s, f}\left(z_{u s, f}-z_{s m, f}\right)+\beta_{s, f}\left(\dot{z}_{u s, f}-\dot{z}_{s m, f}\right)$
$F_{s, z, r}=K_{s, r}\left(z_{u s, r}-z_{s m, r}\right)+\beta_{s, r}\left(\dot{z}_{u s, r}-\dot{z}_{s m, r}\right)$

The free body diagrams of the unsprung masses are drawn in Fig. 6. The longitudinal force balance equation has the same expression for the front and rear unsprung masses, so it is possible to write a general equation for both:

$F_{t, x}-F_{j, x}-m_{u s} \ddot{x}_{u s}=0$

where the longitudinal tyre forces $\left(F_{t, x, f, R}, F_{t, x, f, L}\right.$, $F_{t, x, r, R}$ and $\left.F_{t, x, r, L}\right)$ are computed starting from the tyre delayed torques:

$F_{t, x}=\frac{T_{d t}}{R_{w}}$ 


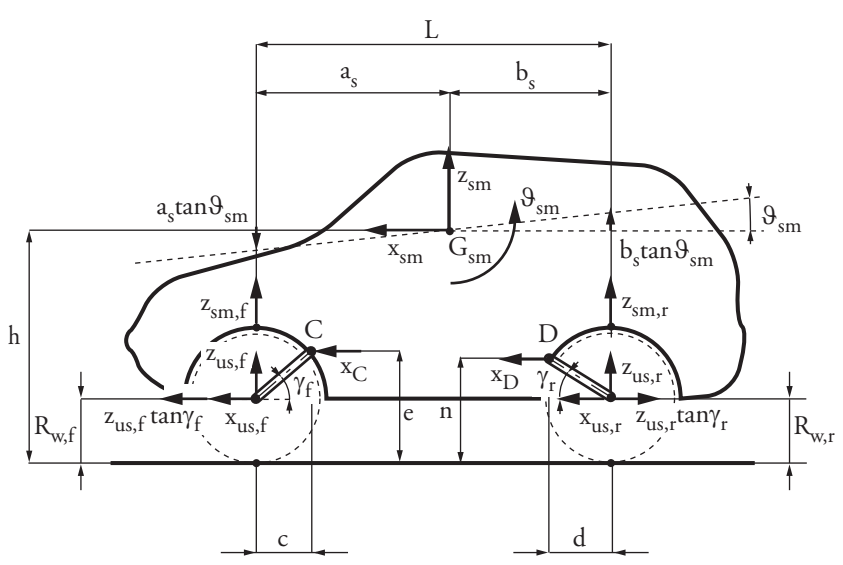

Figure 7: Schematic for the derivation of vehicle kinematic equations

The vertical force balance equation for the unsprung masses is:

$m_{u s} \ddot{z}_{u s}-F_{t, z}+F_{s, z}+F_{j, z}=0$

in which $z_{u s}=R_{w}-R_{w_{0}}$ is the vertical degree of freedom of the unsprung mass, while the vertical tyre forces $\left(F_{t, z, f, R}, F_{t, z, f, L}, F_{t, z, r, R}\right.$ and $\left.F_{t, z, r, L}\right)$ are calculated assuming that the ground is flat without any undulations:

$F_{t, z}=-K_{t, z} z_{u s}-\beta_{t, z} \dot{z}_{u s}$

According to Fig. 6(a) the moment balance equation for the front unsprung mass about point $O$, can be written as follows:

$T_{h s, f}-T_{\text {roll }, f}-m_{u s, f} \ddot{x}_{u s, f} R_{w, f}-F_{j, z, f} c-F_{j_{x, f}} e=0$

Equation (25) is the moment balance about point $Q$ for the rear unsprung mass, according to Fig. 6(b).

$T_{h s, r}-T_{\text {roll }, r}-m_{u s, r} \ddot{x}_{u s, r} R_{w, r}+F_{j, z, r} d-F_{j, x, r} n=0$

To complete the equations that describe the vehicle system, it is necessary to introduce some kinematic relations (see Fig. 7). $z_{s m, f}$ and $z_{s m, r}$ in equation (19) are not state variables, so it is necessary to write the equations considering the link with the chassis vertical displacement $z_{s m}$ and the pitch angle $\vartheta_{s m}$ :

$$
\left\{\begin{array}{l}
\dot{z}_{s m, f}=\dot{z}_{s m}-a_{s} \dot{\vartheta}_{s m} \\
\dot{z}_{s m, r}=\dot{z}_{s m}+b_{s} \dot{\vartheta}_{s m}
\end{array}\right.
$$

The kinematic equations connecting the longitudinal displacement of the wheel centre $x_{u s}$ to its vertical displacement $z_{u s}$ and the longitudinal displacement of the vehicle $x_{s m}$ are:

$$
\left\{\begin{aligned}
\dot{x}_{u s, f} & =\dot{x}_{C}+\dot{z}_{u s, f} \tan \gamma_{f}= \\
& =\dot{x}_{s m}-(h-e) \dot{\vartheta}_{s m}+\left(\frac{e-R_{w, f}}{c}\right) \dot{z}_{u s, f} \\
\dot{x}_{u s, r} & =\dot{x}_{D}-\dot{z}_{u s, r} \tan \gamma_{r}= \\
& =\dot{x}_{s m}-(h-n) \dot{\vartheta}_{s m}-\left(\frac{n-R_{w, r}}{d}\right) \dot{z}_{u s, r}
\end{aligned}\right.
$$

\subsection{Linearised equations}

In order to enable the analysis of the HEV model in the frequency domain, the system equations have to be linearised about specific equilibrium points.

The first non-linear term, that appears in the dynamic equations, is the aerodynamic drag force $F_{a e r}$, that is linearised, using Taylor series truncated at the first order, as in equation (28):

$$
\begin{aligned}
F_{a e r} & =\frac{1}{2} \rho S_{v} C_{d} \dot{x}_{s m}^{2} \Rightarrow \\
F_{a e r, l i n} & =-\frac{1}{2} \rho S_{v} C_{d} \dot{x}_{s m_{0}}^{2}+\rho S_{v} C_{d} \dot{x}_{s m_{0}} \dot{x}_{s m}
\end{aligned}
$$

where: $\rho$ is the air density, $S_{v}$ is the vehicle frontal area, $C_{d}$ is the drag coefficient and $\dot{x}_{s m_{0}}$ is the initial vehicle velocity. The second non-linear term is the rolling resistance torque $T_{\text {roll }}$ that increases quadratically with the wheel speed:

$$
\begin{aligned}
T_{\text {roll }} & =F_{z} R_{w}\left(f_{0}+K R_{w}^{2} \dot{\vartheta}_{w}^{2}\right) \Rightarrow \\
T_{\text {roll }, \text { lin }} & =F_{z_{0}} R_{w}\left(f_{0}-K R_{w}^{2} \dot{\vartheta}_{w_{0}}^{2}+2 K R_{w}^{2} \dot{\vartheta}_{w_{0}} \dot{\vartheta}_{w}\right)
\end{aligned}
$$

where: $f_{0}$ and $K$ are constants, the rolling resistance coefficients and $F_{z}$ is the vertical force between tyre and ground. The wheel torque $T_{t}$ can be computed as the product of the tyre longitudinal force $F_{x}$ and the laden radius $R_{w}$ of the wheel. The steady-state value of the longitudinal tyre force, if the effect of the load transfer and the non-linearities for high longitudinal slip are neglected, can be calculated by multiplying the tyre longitudinal slip stiffness $C_{s}$ (the slope of the linear part of the curve $F_{x}$ vs. longitudinal slip $\sigma$ ), by $\sigma$ :

$T_{t}=R_{w} F_{x}=R_{w} C_{s} \sigma=R_{w} C_{s} \frac{\dot{\vartheta}_{w}-\dot{x}_{u s} / R_{w}}{\dot{\vartheta}_{w}}$

The value of the longitudinal slip stiffness $C_{s}$ introduced in equation (30) can be evaluated from the experimental steady-state characterisation of the tyre or by linearisation of Pacejka Magic Formula ([1], [2] and [9]). 
However, observing the last element of equation (30) where the slip definition is introduced, it can be noted that the approximation of linearity between force and slip, that can be valid for small slip values, does not imply the linearity between this force and the state variables. Indeed the longitudinal slip in traction conditions is the ratio of the speed of the wheel relative to vehicle velocity and the actual tyre speed .

Therefore to obtain a linear expression of the steadystate torque $T_{t}$ is necessary to use the Taylor series for equations with two variables:

$$
\begin{aligned}
& T_{t, l i n}=T_{t}\left(\dot{\vartheta}_{w_{0}}, \dot{x}_{u s_{0}}\right)+\frac{\partial T_{t}}{\partial \dot{\vartheta}_{w}}\left(\dot{\vartheta}_{w_{0}}, \dot{x}_{u s_{0}}\right)\left(\dot{\vartheta}_{w}-\dot{\vartheta}_{w_{0}}\right)+ \\
&+\frac{\partial T_{t}}{\partial \dot{x}_{u s}}\left(\dot{\vartheta}_{w_{0}}, \dot{x}_{u s_{0}}\right)\left(\dot{x}_{u s}-\dot{x}_{u s_{0}}\right) \\
& T_{t, l i n}= \frac{C_{s}}{\dot{\vartheta}_{w_{0}}}\left[\left(R_{w} \dot{\vartheta}_{w_{0}}-\dot{x}_{u s_{0}}\right)+\frac{\dot{x}_{u s_{0}}}{\dot{\vartheta}_{w_{0}}} \dot{\vartheta}_{w}-\dot{x}_{u s}\right] \approx \\
& \approx \beta_{t}\left(\dot{\vartheta}_{w}-\frac{\dot{x}_{u s}}{R_{w}}\right)
\end{aligned}
$$

The last term of equation (31) is obtained using the pure rolling hypothesis applied to the initial condition, i.e. $\dot{x}_{u s_{0}}=R_{w} \dot{\vartheta}_{w_{0}}$.

The equivalent viscous damping coefficient $\beta_{t}\left(=\frac{C_{s} R_{w}^{2}}{\dot{x}_{s m_{0}}}\right)$ that represents the additional damping effect introduced by the steady-state longitudinal behaviour of the tyre tends to infinity when the vehicle velocity tends to zero, consequently this model can be utilised only for speeds sufficiently greater than zero.

In addition, the non-linear elastic and damping behaviour of the clutch damper is linearised and the gear backlash is neglected for both drivetrains.

\subsection{State space formulation}

The model equations presented in the previous sections can be rearranged in the state space formulation:

$\left\{\begin{array}{l}\dot{z}=A \cdot z+B \cdot u \\ y=C \cdot z+D \cdot u\end{array}\right.$

where: $z$ is the state variables vector; $\mathbf{A}$ is the dynamic matrix; $u$ is the external input vector; $\mathbf{B}$ is the input matrix; $y$ is the output vector; $\mathbf{C}$ is the output matrix and $\mathbf{D}$ is the feedthrough matrix.

In the vector $u$ there are the two controllable inputs, engine torque demand $T_{e}$ and electric motor torque demand $T_{m}$, and all the constant terms resulting from the linearisation of the external forces acting on the system, for instance $F_{\text {aer }}$ and $T_{\text {roll }}$.
2.4 Frequency response function selection for drivability evaluation

The matrix $H$, containing all the possible transfer functions between system inputs and outputs, can be derived starting from the system equations in state space:

$H(s)=C\left[(s I-A)^{-1} B\right]+D$

$H$ has $m \times q$ dimension, where $m$ is the number of outputs and $q$ the number of inputs. For each input there are $m$ transfer functions and, more specifically, the element of this matrix in position $(a, b)$ corresponds to the transfer function of the output in position $a$ (vector $y$ ), with respect to the input in position $b$ (vector $u$ ).

Since the HEV powertrains are based on two different actuators, the engine and the electric motor, a suitable transfer function must be selected to represent both effects on the system dynamics. The total torque requested by the driver at the wheels $T_{r e q}$ is the sum of the engine demanded torque evaluated at the front wheels $T_{e}^{*}$ and the electric motor demanded torque evaluated at the rear wheels $T_{m}^{*}$ :

$T_{r e q}=T_{e}^{*}+T_{m}^{*}$

Under the hypothesis of positive actuators power, i.e. considering that the power flow direction is from each motor to the driving wheels, these torques are computed as:

$T_{e}^{*}=i_{d f, f} i_{g, f} \eta_{d f, f} \eta_{g, f} T_{e}$

$T_{m}^{*}=i_{d f, r} i_{g, r} \eta_{d f, r} \eta_{g, r} T_{m}$

Let us consider a fixed torque distribution between the front and the rear axles, with $p$ representing the percentage of the total tractive torque delivered at the front axle by the engine, and $(1-p)$ the electric motor contribution at the rear axle. Under the hypothesis of phase synchronous excitations of the two actuators, the superposition principle can be applied and a transfer function $H_{r e q}$ between vehicle acceleration $\ddot{x}_{s m}$ and torque request $T_{\text {req }}$ can be defined as follows:

$H_{r e q}=\frac{\ddot{x}_{s m}}{T_{r e q}}=p \frac{\ddot{x}_{s m}}{T_{e}^{*}}+(1-p) \frac{\ddot{x}_{s m}}{T_{m}^{*}}$

The previous transfer function characterises the vehicle dynamic response to a driver acceleration request during hybrid operating mode and so it will be adopted to evaluate and compare the drivability performance of this HEV. It is computed starting from the two basic 
transfer functions $H_{e}^{*}$ and $H_{m}^{*}$ that describe the dynamic responses of the purely thermal and purely electric modes:

$H_{r e q}=p s \frac{\dot{x}_{s m}}{T_{e}^{*}}+(1-p) s \frac{\dot{x}_{s m}}{T_{m}^{*}}=p H_{e}^{*}+(1-p) H_{m}^{*}$

\section{Results}

The following section is a summary of the main results obtained throughout the project. In particular the effects of the engaged gear ratios and the torque distribution between the front and rear axles on vehicle drivability are analysed in detail. The comparison of the low frequency drivability response of a conventional frontwheel-driven vehicle and the case study $\mathrm{HEV}$ is dealt with as well.

The transfer functions, between the driver requested torque $T_{r e q}$ and the longitudinal vehicle acceleration $\ddot{x}_{s m}$, are calculated as shown in section 2.4 , with a wheel torque distribution $p$ between the axles equal to $60 \%$. This means that $60 \%$ of the total requested wheel torque is due to the engine torque $T_{e}^{*}$, whilst $40 \%$ of the total requested wheel torque is due to the electric motor torque $T_{m}^{*}$.

The HEV and FWD vehicles here analysed share the same front drivetrain and vehicle data (see data table in the Appendix). Therefore the results in the frequency domain of the two vehicle models are exactly the same if the inertia of the rear driveline components is set to zero. In the time domain, in order to compensate for the different total system inertia, the torque at the electric motor is slightly increased, with respect to the nominal torque distribution of $40 \%$ at the wheels, to provide similar steady-state longitudinal acceleration performance.

\subsection{Effect of the gear ratios on HEV drivability}

Fig. 8 plots the frequency response of the HEV for all the possible combinations of the available gear ratios. This hybrid driveline contains two gearboxes, one for the engine and the other one for the electric drivetrain. The effect of the gear selection on HEV drivability is noticeably evident. The presence of two natural frequencies for each gear combination is clearly visible for the $1^{\text {st }}$ gear in Fig.8(a) and for $1^{\text {st }}$ and $2^{\text {nd }}$ gear in Fig.8(b). The remaining curves relating to the other gear combinations show only one resonance peak because the two natural frequencies of the transmission are very close. One of the two frequencies increases as a function of the front gearbox ratio and combines its effect
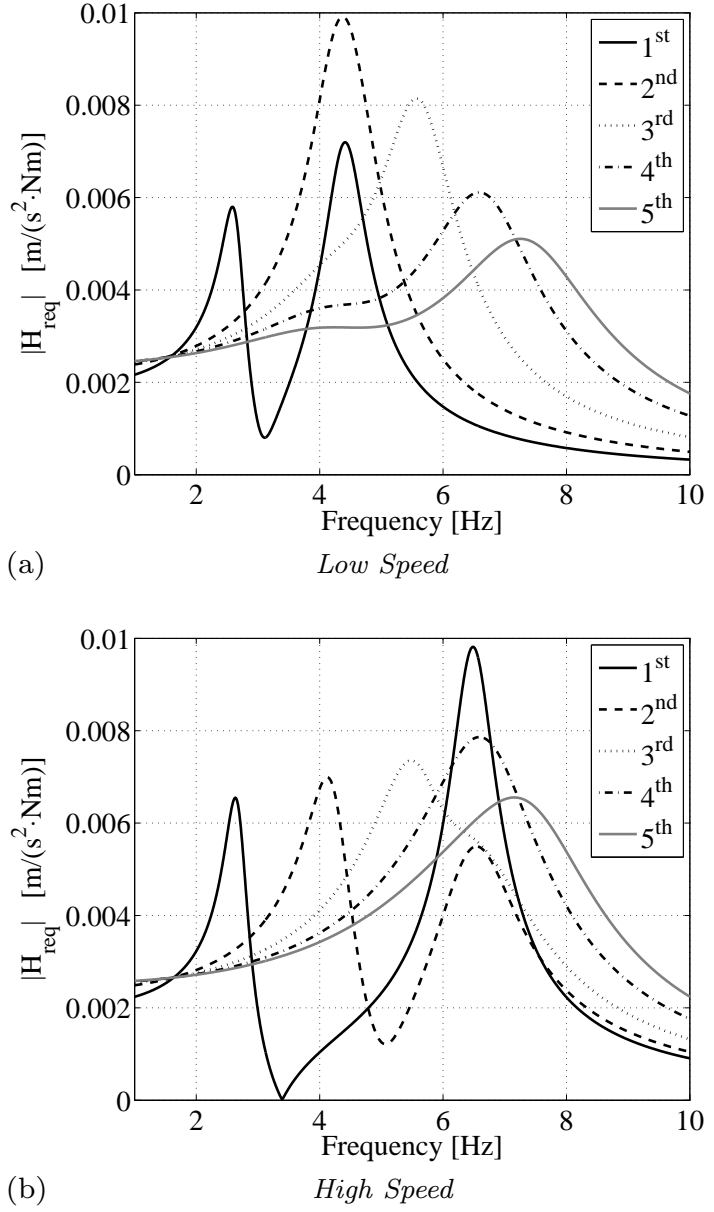

Figure 8: Comparison between HEV frequency response obtained for the two different rear gearbox speeds.

with the other natural frequency that remains constant for a given rear gearbox ratio.

\subsection{Drivability comparison between HEV and FWD}

\subsubsection{Frequency domain}

The comparison of FWD and HEV frequency responses is depicted in Fig. 9. The gear of the rear 2-speed gearbox is selected according to the electric motor torque map, in order to meet the load requirements. More specifically the $1^{\text {st }}$ gear (low speed) of the rear gearbox is engaged for the first two front gearbox speeds and the $2^{\text {nd }}$ (high speed) for the others (see also Table 1 ).

The sharp drop in magnitude of the transfer function (Fig. 9(a)), from the second to the third gear is due to the fact that in this situation the second gear of the electric motor is engaged. 
Table 1: HEV natural frequencies. $\left(\dot{x}_{s m_{0}}\right.$ represents the initial vehicle velocity used for the calculation of the initial conditions and for the linearisation of the motion equations)

\begin{tabular}{|c|c|c|c|c|c|c|}
\hline $\begin{array}{l}\text { Front Gearbox Speed } \\
\text { Rear Gearbox Speed }\end{array}$ & $\begin{array}{l}{[-]} \\
{[-]}\end{array}$ & $\begin{array}{l}1^{\text {st }} \\
1^{\text {st }}\end{array}$ & $\begin{array}{l}2^{\text {nd }} \\
1^{\text {st }}\end{array}$ & $\begin{array}{l}3^{\text {rd }} \\
2^{\text {nd }}\end{array}$ & $\begin{array}{l}4^{\text {th }} \\
2^{\text {nd }}\end{array}$ & $\begin{array}{l}5^{\text {th }} \\
2^{\text {nd }}\end{array}$ \\
\hline$\dot{x}_{s m_{0}}$ & {$\left[\frac{\mathrm{km}}{\mathrm{h}}\right]$} & $\approx 11$ & $\approx 21$ & $\approx 30$ & $\approx 40$ & $\approx 49$ \\
\hline $1^{\text {st }}$ chassis dynamic mode (shaking) & {$[\mathrm{Hz}]$} & 1.11 & 1.11 & 1.11 & 1.11 & 1.11 \\
\hline $2^{\text {nd }}$ chassis dynamic mode (pitching) & [Hz] & 1.67 & 1.67 & 1.67 & 1.67 & 1.67 \\
\hline $1^{\text {st }}$ drivetrain dynamic mode & {$[\mathrm{Hz}]$} & 2.58 & 4.14 & 5.55 & 6.42 & 6.41 \\
\hline $2^{\text {nd }}$ drivetrain dynamic mode & [Hz] & 4.41 & 4.45 & 6.48 & 6.67 & 7.37 \\
\hline
\end{tabular}

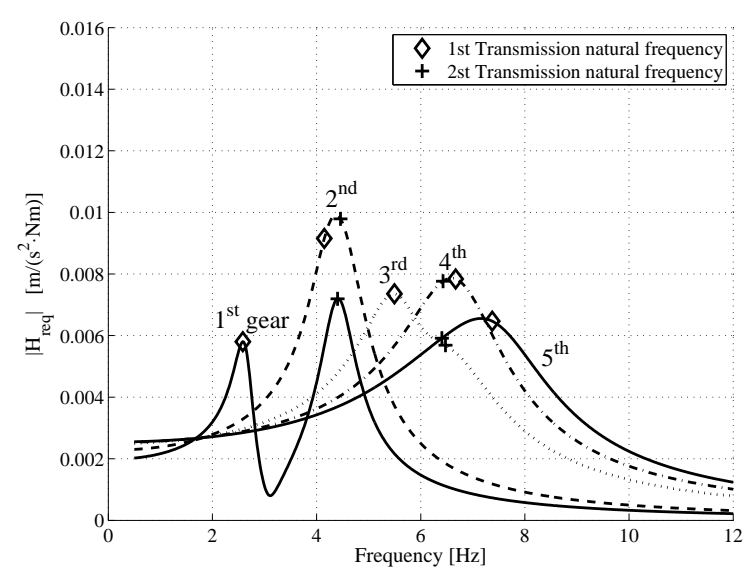

(a)

Frequency response for HEV

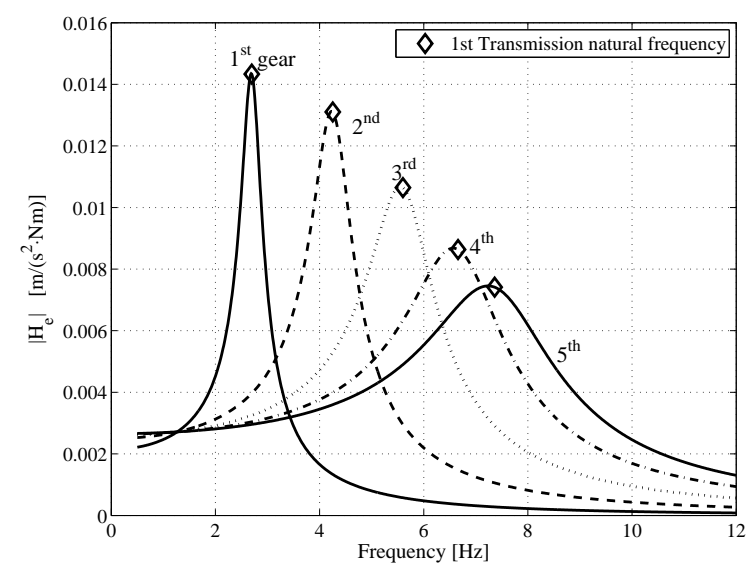

(b)

Frequency response for FWD vehicle

Figure 9: Comparison between the conventional FWD vehicle and the case study through-the-road parallel hybrid electric vehicle in the frequency domain. The plotted frequency response functions are from the wheel torque demand to the vehicle longitudinal acceleration $\left(\left|H_{r e q}\right|=\ddot{x}_{s m} / T_{r e q}\right)$.
In Table 1 the first four natural frequencies of the dynamic system are shown. They are computed as the imaginary part of the eigenvalues of the dynamic matrix $\mathbf{A}$. The first two natural frequencies are in part due to vehicle body modes (vehicle shaking and pitching respectively), while the third and fourth are a result of driveline modes. Looking at the results, it is possible to see, for the second, third, fourth and fifth gear, that the driveline frequencies are very close. This implies that the two peaks merge into a single maximum in the resultant frequency response function (see Fig. 9(a)). By observing the modal shapes at the various natural frequencies it can be concluded that the third natural frequency, for the first, second and third gears (of the front gearbox), is associated with the engine torsional vibration, while, for the fourth and fifth gears, it is due to the electric motor characteristics. The opposite behaviour occurs for the fourth natural frequency, which is associated with the electric motor mode for the first three gears and to the engine mode for the next two gears.

In the lower part of Fig. 9 the Bode diagram of the response of a traditional FWD vehicle is drawn: there is only one peak for each gear in this frequency range, and the values of the natural frequencies experience a decrease as a function of the gear ratios. It is therefore interesting to note that the addition of the electric motor on the rear axle introduces a new natural frequency, not very different from the one associated to the fully thermal operating mode (i.e. engine only mode). Since frequencies between 1 and $10 \mathrm{~Hz}$ are the most significant for comfort, according to [3], [6] and [12], this phenomenon must be taken into account during the set-up of the driveline, if the aim is to reach a satisfactory trade-off between quick response and comfort. However it must be noted that the amplitude of the frequency response function is always lower in the hybrid configuration and as a consequence the hybrid vehicle seems to be more comfortable. The higher static gain of the response for higher gears is due to the lower equivalent inertia of the system in those operating conditions. 


\subsubsection{Time domain}

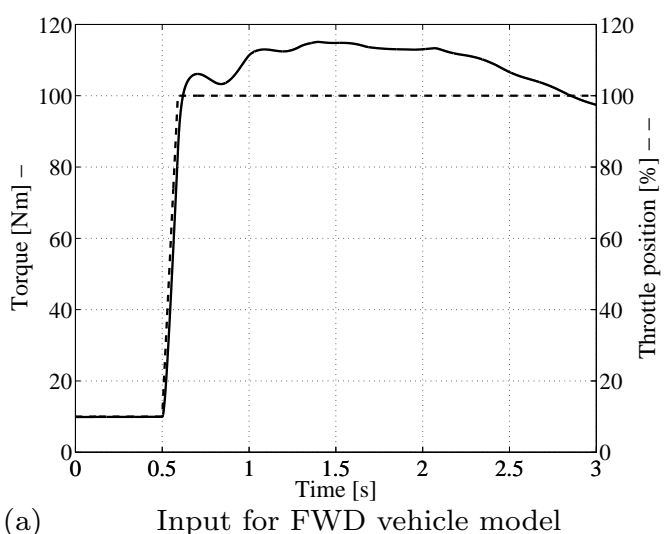

(a)

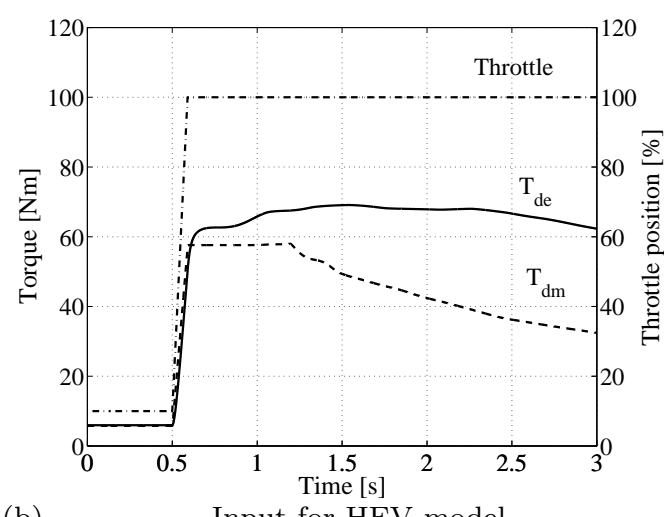

(b)

Input for HEV model

Figure 10: (a) Left axis: $T_{d e}$ the engine delayed torque of FWD vehicle; Right axis: throttle position. (b) Left axis: $T_{d e}$ the engine delayed torque of $\mathrm{HEV}$ vehicle; Right axis: throttle position.

Vehicle longitudinal acceleration is chosen as the benchmark for the comparisons because it represents one of the key factors when assessing drivability during tip-in tests.

Fig.10(a) and (b) shows the input torques imposed during the tip-in test for the FWD and HEV respectively. Regarding the HEV, while the engine torque is substantially constant after the step, that occurs at 0.5 $\mathrm{s}$ of simulation time, the electric motor torque starts decreasing hyperbolically with time after $\approx 1.25 \mathrm{~s}$, according to its constant power region. The engine torque characteristic for the $\mathrm{HEV}$ is set to provide $60 \%$ of the maximum torque the engine can deliver in the FWD vehicle, while the electric motor torque is specified in order to provide the same initial acceleration as the FWD vehicle during the tip-in test. Moreover the first gear is imposed at the front gearbox because that is a

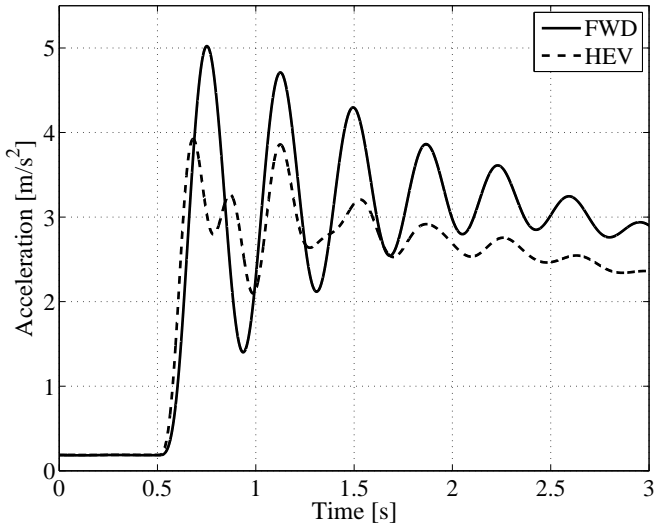

Figure 11: Comparison between FWD and HEV during tip-in test.

critical condition for FWD vehicle comfort.

If the time history of the HEV acceleration is compared with that of the conventional FWD vehicle (see Fig. 11), it is possible to observe, also in this graph, the presence of two frequencies, one about twice the other. In case of the hybrid vehicle the presence of two different paths through the driveline generates two natural frequencies, one for each axle, as per section 3.2.

In addition, while for a conventional FWD vehicle, according to [5], we can consider the oscillations of the acceleration as a second order response, for the hybrid vehicle at least a fourth order is needed, particularly in cases where there are two distinct resonance peaks on the Bode diagram, e.g. the first gear.

\subsection{Comparison of the models}

The comparison between the models presented in section 2 is performed in the time domain, using the tip-in test as a reference manoeuvre. In Fig. 12 the comparison in terms of longitudinal acceleration between the models is shown. The figure illustrates that the simple model approximates the longitudinal acceleration trend without any discernible difference from the other more complex models. This observation allows us to conclude that further model complexity, by introducing the halfshafts or the open differential model, is unnecessary when assessing drivability because the improvements on the obtained results are not visible. Even the model with the sprung mass dynamics is not significantly different, in terms of vehicle acceleration response, from the open differential model; this happens because the added DOFs of this model relating to chassis dynamics are practically decoupled from the driveline torsional vibration modes. However, the model with the sprung mass dynamics allows the study of vehicle pitch and 


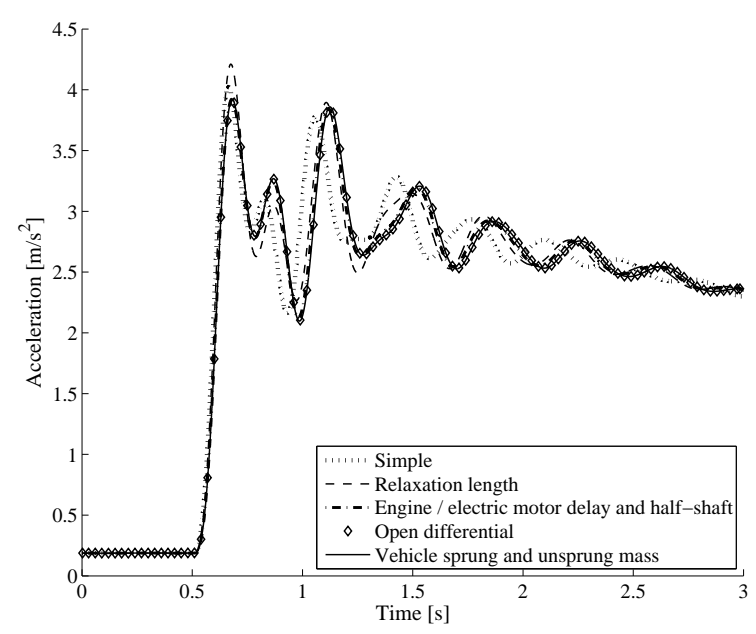

Figure 12: Comparison of the time histories of longitudinal acceleration for the different hybrid vehicle models ( $1^{\text {st }}$ gear for the front powertrain).

shake motions that are other crucial factors for a comprehensive comfort assessment.

3.4 Influence of the torque distribution between the front and rear axles

The distribution of the driving torques between the axles modifies the dynamic response of the HEV. As can be seen in Fig. 13, if the first gear is selected for the front and rear gearbox, the amplitude of the second peak increases as a function of the percentage $p$ of torque demand from the electric motor.

During normal HEV driving and energy management operation, $p$ is usually low at low speed and the most significant mode is at 4-5 Hz. Then at higher speeds $p$ is usually higher, but the engine drivetrain is in second or third gear and thus has a higher frequency peak. As a consequence, the important conclusion is that there may never be a significant drivetrain response peak below $4 \mathrm{~Hz}$ in this HEV layout. The low frequency peak below $4 \mathrm{~Hz}$ is a major drivability issue of internal combustion engine driven vehicles.

\section{Conclusions}

The following conclusions can be drawn from the research presented in this paper:

- Depending on the engaged gear ratios of the front and rear drivetrains, the frequency response of the physical parameters relevant to HEV drivability can be characterised by either one or two peaks, one for each axle, in the range 1-10 $\mathrm{Hz}$.

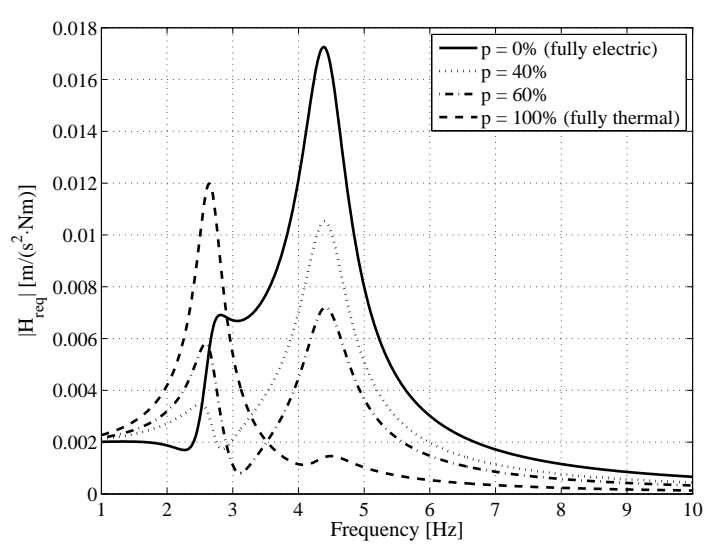

Figure 13: Torque distribution effect between the thermal (front) and electric (rear) axles on the frequency response of the HEV. The lowest gear is imposed at both front and rear gearboxes.

- In opposition to the typical second order response of FWD internal combustion engine driven vehicles, the HEV tip-in response shows a more complex time history due to the combination of the first natural frequencies of each drivetrain.

- In order to achieve a good approximation of HEV drivability response, it is necessary to consider the linear torsion dynamics of half-shafts and tyres. More complex models can be useful for component analysis and design but do not significantly affect the overall response.

- The front-to-rear torque bias ratio exerts a very relevant effect on the low frequency drivability of HEVs.

The effect of the powertrain mounting system needs to be evaluated in the future steps of this project.

\section{References}

1. E. Bakker, L. Nyborg and H.B. Pacejka (1987), 'Tyre Modelling for Use in Vehicle Dynamics Studies', SAE 870421, Paper presented at the SAE International Congress and Exhibition. February 23-27, 1987. Detroit (MI), USA.

2. E. Bakker, H.B. Pacejka and L.Lidner (1989), 'A New Tire Model with an Application in Vehicle Dynamics Studies', SAE Technical Paper, Number: 890087.

3. J. Baumann, A. Swarnakar, U. Kiencke, T. Schlegl (2005), 'A Robust Controller Design for Anti-Jerking', SAE 2005-01-0041, Paper presented at the SAE International Congress and Exhibition. April, 2005. Detroit (MI), USA.

4. Y.C. Choi, H.B. Song, J.H. Lee and H.S. Cho (2005), 'An Experimental Study for Drivability Improvements in Vehicle Acceleration Mode', Proceedings of the Institution of Mechanical Engineers, Part D: Journal of Automobile Engineering, Vol.217, No.7, pp.623-631, 2005. 
5. R.E. Dorey, C.B. Holmes (1999), 'Vehicle Driveability Its Characterisation and Measurement', SAE 1999-010949, Paper presented at the SAE International Congress and Exhibition. March, 1999. Detroit (MI), USA.

6. J. Fredriksson (2006), 'Improved Drivability of a Hybrid Electric Vehicle Using Powertrain Control', Int. J. of Alternative Propulsion 2006, Vol.1, No.1, pp.97-111.

7. B.S. Kim, J.H. Kim and S. II Kim (2008), 'Vehicle Drift Investigation During Straight Line Acceleration and Braking', SAE 2008-01-0588, Paper presented at the SAE International Congress and Exhibition. April, 2008. Detroit (MI), USA.

8. S. Kim, J. Park, J. Hong, M. Lee and H. Sim (2009), 'Transient Control Strategy of Hybrid Electric Vehicle during Mode Change', SAE 2009-01-0228, Paper presented at the SAE International Congress and Exhibition. April, 2009. Detroit (MI), USA.

9. H.B. Pacejka (2006), Tyre and Vehicle Dynamics, Butterworth-Heinemann.

10. J. Reimpell, H. Stoll and J.W. Betzler (2001), The automotive chassis - Engineering principles, Second edition, SAE International and Edward Arnold Publishing/Bookpoint.

11. A. Sorniotti, E. Galvagno, A. Morgando and M. Velardocchia (2007), 'An Objective Evaluation of the Comfort During the Gear Shift Process', SAE 2007-01-1584, Paper presented at the SAE International Congress and Exhibition. April, 200\%. Detroit (MI), USA.

12. A. Sorniotti (2008), 'Driveline Modeling, Experimental Validation and Evaluation of the Influence of the Different Parameters on the Overall System Dynamics', SAE 2008-01-0632, Paper presented at the SAE International Congress and Exhibition. April, 2008. Detroit (MI), USA.

13. D.D. Torkzadeh, J. Baumann and U. Kiencke (2003), 'A Neuro-Fuzzy Approach for Anti-Jerk Control', SAE 200301-0361, Paper Presented at the SAE International Congress and Exhibition. March, 2003. Detroit (MI), USA.

14. J. Zhang, X. Lu, L. Wang, S. Chen and S. Li (2008), 'A Study on the Drivability of Hybrid Electric Vehicles', SAE 2008-01-1572, Paper presented at the SAE International Powertrains, Fuels and Lubricants Congress, June, 2008. Shanghai, China.

\section{Appendix: Model Parameters}

\begin{tabular}{|c|c|c|}
\hline Description & Value & Unit \\
\hline \multicolumn{3}{|c|}{ Vehicle } \\
\hline$m_{s m}$ & 1030 & $\mathrm{~kg}$ \\
\hline$m_{u s, f}=m_{u s, r}$ & 50 & $\mathrm{~kg}$ \\
\hline$C_{d}$ & 0.32 & - \\
\hline$S_{v}$ & 2.04 & $\mathrm{~m}^{2}$ \\
\hline$\rho$ & 1.204 & $\mathrm{~kg} / \mathrm{m}^{3}$ \\
\hline$a_{s}$ & 0.890 & $\mathrm{~m}$ \\
\hline$b_{s}$ & 1.620 & $\mathrm{~m}$ \\
\hline \multicolumn{3}{|c|}{ Electric Motor } \\
\hline Maximum Power & 19.3 & $\mathrm{~kW}$ \\
\hline Maximum Torque & 82 & $\mathrm{Nm}$ \\
\hline Base Speed & 2200 & $\mathrm{rpm}$ \\
\hline Maximum Speed & 8000 & rpm \\
\hline$J_{m}$ & 0.09 & $\mathrm{kgm}^{2}$ \\
\hline$\tau_{m}$ & 0.0013 & $\mathrm{~s}$ \\
\hline \multicolumn{3}{|c|}{ Gasoline Engine (FWD) } \\
\hline Peak Power & 58 at $6000 \mathrm{rpm}$ & $\mathrm{kW}$ \\
\hline Peak Torque & 115 at $3300 \mathrm{rpm}$ & $\mathrm{Nm}$ \\
\hline$J_{e}$ & 0.115 & $\mathrm{kgm}^{2}$ \\
\hline$\tau_{e}$ & $2.7 / \dot{\vartheta}_{e}$ & $\mathrm{~s}$ \\
\hline \multicolumn{3}{|c|}{ Front Driveline } \\
\hline$J_{c}$ & 0.020 & $\mathrm{kgm}^{2}$ \\
\hline$K_{c d}$ & 573.0 & $\mathrm{Nm} / \mathrm{rad}$ \\
\hline$\beta_{c d}$ & 4.900 & $\mathrm{Nms} / \mathrm{rad}$ \\
\hline$i_{g, f}[1 \mathrm{st} \ldots 5 \mathrm{th}]$ & {$\left[\begin{array}{llllll}3.91 & 2.16 & 1.48 & 1.12 & 0.92\end{array}\right]$} & - \\
\hline$i_{d f, f}$ & 3.73 & - \\
\hline$\eta_{g, f}$ & 0.98 & - \\
\hline$\eta_{d f, f}$ & 0.98 & - \\
\hline$K_{h s, f l}$ & 4800 & $\mathrm{Nm} / \mathrm{rad}$ \\
\hline$K_{h s, f r}$ & 3200 & $\mathrm{Nm} / \mathrm{rad}$ \\
\hline$J_{d f, f}$ & 0.065 & $\mathrm{kgm}^{2}$ \\
\hline \multicolumn{3}{|c|}{ Rear Driveline } \\
\hline$i_{g, r}[$ low high $]$ & {$\left[\begin{array}{ll}3 & 2\end{array}\right]$} & - \\
\hline$i_{d f, r}$ & 3.7 & - \\
\hline$\eta_{g, r}$ & 0.98 & - \\
\hline$\eta_{d f, r}$ & 0.98 & - \\
\hline$K_{h s, r l}$ & 5800 & $\mathrm{Nm} / \mathrm{rad}$ \\
\hline$K_{h s, r r}$ & 4260 & $\mathrm{Nm} / \mathrm{rad}$ \\
\hline$J_{d f, r}$ & 0.065 & $\mathrm{kgm}^{2}$ \\
\hline \multicolumn{3}{|c|}{ Tyre } \\
\hline$R_{w}$ & 0.294 & $\mathrm{~m}$ \\
\hline$J_{w}$ (single) & 0.695 & $\mathrm{kgm}^{2}$ \\
\hline$f_{0}$ & 0.0142 & - \\
\hline$K$ & $9.033 \mathrm{e}-006$ & $(\mathrm{~s} / \mathrm{m})^{2}$ \\
\hline$K_{t, z}$ & 200000 & $\mathrm{~N} / \mathrm{m}$ \\
\hline$\beta_{t, z}$ & 1000 & $\mathrm{Ns} / \mathrm{m}$ \\
\hline$C_{s, f}$ & 51000 & $\mathrm{~N}$ \\
\hline$C_{s, r}$ & 34000 & $\mathrm{~N}$ \\
\hline$L_{r, f}=L_{t, r}$ & 0.15 & $\mathrm{~m}$ \\
\hline \multicolumn{3}{|c|}{ Suspension System } \\
\hline$K_{s, f}$ & 16500 & $\mathrm{~N} / \mathrm{m}$ \\
\hline$K_{s, r}$ & 17000 & $\mathrm{~N} / \mathrm{m}$ \\
\hline$\beta_{s, f}$ & 1220 & $\mathrm{Ns} / \mathrm{m}$ \\
\hline$\beta_{s, r}$ & 1260 & $\mathrm{Ns} / \mathrm{m}$ \\
\hline
\end{tabular}

